\title{
NONCOMMUTATIVE TOPOLOGICAL DYNAMICS. II
}

BY

DANIEL AVITZOUR

\begin{abstract}
This part deals with almost periodic and weakly mixing $C^{*}$-flows, and with disjointness and weak disjointness of $C^{*}$-flows (flows on $C^{*}$-algebras). The main result is a generalization to $C^{*}$-flows of Keynes and Robertson's characterization of minimal weakly mixing flows. Examples are discussed exhibiting anomalous behaviour of disjointness in the $C^{*}$-flow case.
\end{abstract}

0. Introduction. In this paper we shall continue the attempt, started in [1], to generalize topological dynamics to the context of $C^{*}$-flows $(G, A)$ where $G$ is a group of *-automorphisms of a (unital) $C^{*}$-algebra $A$.

The main positive result in this paper is a characterization of minimal weakly mixing $C^{*}$-flows as the "extreme opposite of almost periodic flows", generalizing a result of Keynes and Robertson in topological dynamics.

In $§ 1$ I review almost periodic $C^{*}$-flows, defined in [5, 9]. I discuss the relationship of almost periodicity to ergodicity and minimality, defined in the preceding paper [1]. I also show that almost periodic $C^{*}$-flows are semisimple (pointwise almost periodic).

In $§ 2$ I define weak mixing and weak disjointness and several examples are given. Weak mixing is first discussed for $W^{*}$-flows to provide motivation for the definition in the $C^{*}$-flow case.

The main result, mentioned above, is proven in $\$ 4$ after some technical preparation in $\$ 3$.

In $\$ 5$ I briefly discuss disjointness for $C^{*}$-flows and I give an example showing the anomalous behaviour of this concept: a $C^{*}$-flow may be disjoint from all minimal $C^{*}$-flows including itself.

The reader is referred to [1] for notations and conventions and for the definitions of ergodicity and minimality in the $C^{*}$-flow case. As in [1] all $C^{*}$-algebras are assumed to be unital.

\section{Almost periodicity.}

1.0. Definition. A $C^{*}$-flow $(G, A)$ is almost periodic if for all $a \in A$ the orbit $G a$ is relatively compact in norm topology.

1.1. It is shown in $[5,9]$, that the definition is equivalent to each of the following conditions:

(1) $G$ acts equicontinuously on the states of $A$.

Received by the editors August 18, 1981 and, in revised form, December 22, 1982.

1980 Mathematics Subject Classification. Primary 46L55; Secondary 46L05. 
(2) $G$ is relatively compact in $A^{A}$ in the product topology.

(3) The closure of $G$ in $\operatorname{Aut}(A)$, with pointwise convergence topology, is a compact group.

In view of (3) there is a 1-1 correspondence between almost periodic $G$-flows and all $\tilde{G}$-flows, where $\tilde{G}$ is the maximal compactification of $G$ (see e.g. [13, p. 296] for the definition of $\tilde{G}$ ). By [1, 3.5 and 4.4$]$ this correspondence respects ergodicity and minimality. It follows that the study of almost periodc $C^{*}$-flows is equivalent to the study of $C^{*}$-flows with a compact group.

1.2. Definition. Let $(G, A)$ be a $C^{*}$-flow.

$$
A_{\text {ap }}=\{a \in A: G a \text { relatively compact }\} \text {. }
$$

As shown in [9, p. 198], $A_{\text {ap }}$ is a $G$-invariant subalgebra, so $\left(G, A_{\text {ap }}\right)$ is the biggest almost periodic factor of $(G, A)$.

1.3. Proposition. Almost periodicity passes to factors, opposites, inductive limits and tensor products.

Proof. Factors and opposites. Immediate.

Tensor products. Let $(G, A)$ and $(G, B)$ be almost periodic $C^{*}$-flows, and let $A \otimes_{\alpha} B$ be some $C^{*}$-tensor product of $A$ and $B . G$ acts on $A \otimes_{\alpha} B$ by the diagonal action: $s(a \otimes b)=s a \otimes s b$. Now, if $a \in A$ and $b \in B, G(a \otimes b) \subseteq G a \otimes G b=$ relatively compact, so $a \otimes b \in\left(A \otimes_{\alpha} B\right)_{\text {ap }}$. Since elementary tensors span $A \otimes_{\alpha} B$, we get $A \otimes_{\alpha} B=\left(A \otimes_{\alpha} B\right)_{\text {ap }}$.

Inductive limits. Let $(G, A)$ be a $C^{*}$-flow, and let $\left(G, A_{n}\right)$ be increasing factors such that $A=\left(\cup A_{n}\right)^{-}$. If $\left(G, A_{n}\right)$ are almost periodic, then $A_{n} \subseteq A_{\text {ap }}$, so $A=$ $\left(\cup A_{n}\right)^{-}=A_{\text {ap }}$. Q.E.D.

1.4. Proposition. Let $(G, A)$ be an almost periodic $C^{*}$-flow. The following are equivalent:

(1) $(G, A)$ is minimal.

(2) $(G, A)$ is ergodic.

(3) $A_{G}=\mathbf{C}$.

(4) $(G, A)$ is strictly ergodic.

(5) $(G, A)$ is uniquely ergodic.

(6) For any covariant representation $(u, \pi)$ of $(G, A)$, the $W^{*}$-flow $\left(G, \pi(A)^{\prime \prime}\right)$ is ergodic.

Proof. Without limiting generality we may assume that $G$ is compact.

$(1) \Rightarrow(2) \Rightarrow(3)$. From definitions.

(3) $\Rightarrow(4)$. Define for $a \in A, \phi(a)=\int_{G}(s a) d s \in A_{G}=$ C. $\phi$ is a faithful $G$ invariant state on $A$. Let $\psi$ be any $G$-invariant state on $A$. For $a \in A$,

$$
\psi(a)=\int_{G} \psi(s a) d s=\psi\left(\int_{G}(s a) d s\right)=\psi(\phi(a))=\phi(a) .
$$

So $(G, A)$ is strictly ergodic.

(4) $\Rightarrow(5)$. From definitions. 
(5) $\Rightarrow(3)$. Let $\phi$ be the unique $G$-invariant state on $A$. Let $\psi$ be a state on $A$. The state $a \rightarrow \int_{G} \psi(s a) d s$ is $G$-invariant, so equal to $\phi$, that is $\phi(a)=\int_{G} \psi(s a) d s$. In particular, for $a \in A_{G}, \phi(a)=\psi(a)$, so $a$ is constant on $K$.

(4) $\Rightarrow(1)$. By $[1,4.5]$. Note that $G$ is compact, hence amenable.

$(6) \Rightarrow(2)$. Take $(u, \pi)$ such that $\pi$ is faithful and use $[1,3.4]$.

(3) $\Rightarrow(6)$. Let $(u, \pi)$ be a covariant representation of $(G, A)$ on a Hilbert space $H$.

Let $P$ be the $\sigma$-weakly continuous, conditional expectation on $B(H)$,

$$
P(T)=\int_{G} u(s) T u(s)^{*} d s .
$$

Since $A_{G}=\mathbf{C}$, we have $P(\pi(A))=\mathbf{C}$, hence $P\left(\pi(A)^{\prime \prime}\right)=\mathbf{C}$, so $\left(\pi(A)^{\prime \prime}\right)_{G}=\mathbf{C}$. Q.E.D.

1.5. In [10] all $C^{*}$ flows $(G, A)$, with $G$ compact Abelian, and $A_{G}=\mathbf{C}$, were described. By 1.4 this is equivalent to finding all minimal almost periodic $C^{*}$-flows with an Abelian group.

\subsection{Definition. A $C^{*}$-flow $(G, A)$ is semisimple if}

$$
1=\sup \{q: q \text { minimal nonzero, } G \text {-invariant, closed projection }\} ;
$$

equivalently if

$$
0=\cap\{\vec{J}: J \text { maximal proper, } G \text {-invariant, hereditary subalgebra }\},
$$

where $\bar{J}$ is the $\sigma$-weak closure of $J$ in $A^{* *}$. The equivalence follows from the fact that if $p$ is an open projection and $J$ is the corresponding hereditary subalgebra, then $\bar{J}=p A^{* *} p$. The first condition reduces in the commutative case to the usual one (see e.g. [4]). The term pointwise almost periodic is used in older texts.

1.7. Proposition. Let $(G, A)$ be an almost periodic $C^{*}-$ flow. The map $J \rightarrow J \cap A_{G}$ is a 1-1 correspondence between the $G$-invariant, hereditary subalgebras of $A$, and the hereditary subalgebras of $A_{G}$.

Proof. It is more convenient to work with norm-closed order ideals in $A$, rather than hereditary subalgebras.

Without limiting generality assume that $G$ is compact and let $P: A \rightarrow A_{G}$ be the conditional expectation $P(a)=\int_{G}(s a) d s$. If $j \subseteq A_{G}$ is an order ideal, then $P^{-1}(j)^{+}$ is a $G$-invariant order ideal in $A$. I want to show that the map $j \rightarrow P^{-1}(j)^{+}$is the inverse of the map $J \rightarrow J \cap A_{G}$.

If $j$ is an order ideal of $A_{G}$ it is clear that $P^{-1}(j)^{+} \cap A_{G}=j$.

On the other hand, let $J$ be a $G$-invariant order ideal of $A$. Immediately: $J \subseteq P^{-1}\left(J \cap A_{G}\right)^{+}$. For the reverse inclusion write

$$
J=\bigcap\left\{J_{s \phi}: s \in G, \phi \in I\right\},
$$

where $I$ is a set of states, and for any $\phi, J_{\phi}=(\operatorname{ker} \phi)^{+}$. If $a \in P^{-1}\left(J \cap A_{G}\right)^{+}$then $P(a) \in J_{\phi}$ for all $\phi \in I$, that is $\int_{G} \phi(s a) d s=0$, so $\phi(s a)=0$ for all $s \in G$. Hence

$$
a \in \cap\left\{J_{s \phi}: s \in G, \phi \in I\right\}=J \text {. Q.E.D. }
$$

1.8. Proposition. Every almost periodic $C^{*}$-flow is semisimple. 
Proof. Let $(G, A)$ be an almost periodic $C^{*}$-flow. As usual, assume that $G$ is compact. Note that the conditional expectation $P$ defined in the proof of 1.7 extends to a $\sigma$-weakly continuous, conditional expectation of $A^{* *}$ onto $\left(A^{* *}\right)_{G}$, defined by the same formula.

Identifying $\left(A_{G}\right)^{* *}$ with a subspace of $A^{* *}$, we have

$$
\left(A^{* *}\right)_{G}=P\left(A^{* *}\right)=(P(A))^{-}=\left(A_{G}\right)^{-}=\left(A_{G}\right)^{* *} .
$$

If $J$ is a $G$-invariant, hereditary subalgebra of $A, P J \subseteq J$ so $P(\bar{J}) \subseteq \bar{J}$. Hence $\cap\{\bar{J}: J$ maximal, $G$-invariant, hereditary subalgebra $\} \cap A_{G}^{* *}=P(\cap\{\bar{J}: J$ maximal, $G$-invariant, hereditary subalgebra $\}$ ). Since $P$ is faithful, it is enough to show that $\cap\{\bar{J}: J$ maximal, $G$-invariant, hereditary sublgebra $\} \cap A_{G}^{* *}=\{0\}$. Now $\bar{J} \cap$ $A_{G}^{* *}=P(\bar{J})=P(J)^{-}=\left(J \cap A_{G}\right)^{-}$, so $\cap\{\bar{J}: J$ maximal, $G$-invariant, hereditary subalgebra $\} \cap A_{G}^{* *}=\cap\left\{\left(J \cap A_{G}\right)^{-}: J\right.$ maximal, $G$-invariant, hereditary subalgebra $\}=\bigcap\left\{\bar{j}: j\right.$ maximal hereditary subalgebra of $\left.A_{G}\right\}=0$, where the equality before last follows from 1.7. Q.E.D.

1.9. If $A=\tilde{K}(H)$ (compact operators on $H$ with unit adjoined), then 1.8 says exactly that a projective representation of a compact group is a direct sum of irreducible representations (see [1, 3.7] for discussion of flows on $\tilde{K}(H)$ ).

\section{Weak mixing and weak disjointness.}

2.0. I shall first deal with weak mixing in $W^{*}$-flows. This has so far been discussed only in terms of the "probabilistic" definition of weak mixing (2.2) which does not allow for generalization to topological dynamics. I shall show that it is equivalent to a definition based on the tensor product of the flow with itself which can be generalized to $C^{*}$-flows.

First I need a remark about the opposite $A^{\text {op }}$ of a $C^{*}$ (or $W^{*}$ ) algebra $A$ defined in $[1,2.5]$.

2.1. The representations of $A, A^{\text {op }}$ are closely connected. If $\pi$ is a representation of $A$ on a Hilbert space $H, A^{\text {op }}$ acts on the conjugate Hilbert space $\bar{H}$ by

$$
\pi^{\mathrm{op}}(a) \bar{\xi}=\overline{\pi\left(a^{*}\right) \xi}, \quad a \in A, \xi \in H .
$$

We get a 1-1 correspondence between the representations of $A$ and $A^{\mathrm{op}}$. If $\phi$ is a state of $A$ (also a state of $A^{\mathrm{op}}$ ) the GNS representations of $A$ and $A^{\mathrm{op}}$ corresponding to $\phi$ are connected by the map $\pi \rightarrow \pi^{\text {op }}$.

2.2. Definition. Let $(G, M)$ be a $W^{*}$-flow where $G$ is locally compact. A $G$-invariant normal state $\phi$ on $M$ is weakly mixing if

$$
\underline{M}\{|\phi(s a \cdot b)-\phi(a) \phi(b)|: s \in G\}=0
$$

for all $a, b$ in a dense subspace of $M$. Here $\underline{M}$ is the invariant mean on weakly almost periodic functions (see e.g., [6, p. 75] for this definition and the properties of $\underline{M}$ ).

In the case $M=L^{\infty}(X, \mu), \phi=\mu$, this is the usual definition in ergodic theory.

2.3. Proposition. Let $\left(G, M_{i}\right)$ be $W^{*}$-flows and $\phi_{i}$ weakly mixing states on them, $i=1,2$. Then $\phi_{1} \otimes \phi_{2}$ is weakly mixing on $\left(G, M_{1} \otimes M_{2}\right)$.

Proof. It is enough to check the definition with two elementary tensors in $M_{1} \otimes M_{2}$. 


$$
\begin{aligned}
\underline{M}\left(\mid\left(\phi_{1} \otimes\right.\right. & \left.\left.\phi_{2}\right)\left(s\left(a_{1} \otimes a_{2}\right) \cdot b_{1} \otimes b_{2}\right)-\left(\phi_{1} \otimes \phi_{2}\right)\left(a_{1} \otimes a_{2}\right)\left(\phi_{1} \otimes \phi_{2}\right)\left(b_{1} \otimes b_{2}\right) \mid\right) \\
\leqslant & \underline{M}\left(\|\left[\phi_{1}\left(s a_{1} \cdot b_{1}\right)-\phi_{1}\left(a_{1}\right) \phi_{1}\left(b_{1}\right)\right] \phi_{2}\left(a_{2}\right) \phi_{2}\left(b_{2}\right) \mid\right) \\
& +\underline{M}\left(\left|\phi_{1}\left(s a_{1} \cdot b_{1}\right)\left[\phi_{2}\left(s a_{2} \cdot b_{2}\right)-\phi_{2}\left(a_{2}\right) \phi_{2}\left(b_{2}\right)\right]\right|\right) \\
\leqslant & \left\|a_{2}\right\|\left\|b_{2}\right\| \underline{M}\left(\left|\phi_{1}\left(s a_{1} \cdot b_{1}\right)-\phi_{1}\left(a_{1}\right) \phi_{1}\left(b_{1}\right)\right|\right) \\
& +\left\|a_{1}\right\|\left\|b_{1}\right\| \underline{M}\left(\left|\phi_{2}\left(s a_{2} \cdot b_{2}\right)-\phi_{2}\left(a_{2}\right) \phi_{2}\left(b_{2}\right)\right|\right)=0 . \quad \text { Q.E.D. }
\end{aligned}
$$

2.4. Proposition. Let $(G, M)$ be a $W^{*}$-flow and $\phi$ a $G$-invariant, normal, faithful state. The following are equivalent:

(1) $\phi$ is weakly mixing.

(2) $\phi \otimes \phi$ is weakly mixing for $\left(G, M^{\mathrm{op}} \otimes M\right)$.

(3) $\left(G, M^{\mathrm{op}} \otimes M\right)$ is ergodic.

$\left(2^{\prime}\right) \phi \otimes \phi$ is weakly mixing for $(G, M \otimes M)$.

$\left(3^{\prime}\right)(G, M \otimes M)$ is ergodic.

And they imply

(4) $(G, M)$ is ergodic.

Proof. (1) $\Rightarrow(2)$. By 2.3 .

(1) $\Rightarrow\left(2^{\prime}\right)$. Same.

$(1) \Rightarrow(4)$. Let $p$ be a $G$-invariant projection in $M$.

$$
\left|\phi(p)-\phi(p)^{2}\right|=\underline{M}(|\phi(s p \cdot p)-\phi(p) \phi(p)|)=0,
$$

so $\phi(p)=0$ or 1 . Since $\phi$ is faithful, $p=0$ or 1 .

$(2) \Rightarrow(3)$. Follows from (1) $\Rightarrow$ (4) where $M$ is replaced by $M^{\mathrm{op}} \otimes M$ and $\phi$ by $\phi \otimes \phi$, which is faithful.

$\left(2^{\prime}\right) \Rightarrow\left(3^{\prime}\right)$. Similarly.

(3) $\Rightarrow(1)$. We can assume that $M \subseteq B(H)$ where $H$ is a Hilbert space on which $G$ acts by a unitary representation $u$, and $\phi$ corresponds to a cyclic and separating unit vector $\omega \in H$, which is $G$-invariant. $M^{\text {op }} \otimes M$ acts naturally on $\bar{H} \otimes H$. Since $\left(G, M^{\mathrm{op}} \otimes M\right)$ is ergodic, $\bar{\omega} \otimes \omega$ is the only $G$-invariant vector in $\bar{H} \otimes H$, up to scalar multiples (see [6, p. 77, Théorème VI.2]).

This implies, by elementary properties of $\underline{M}$,

$$
\underline{M}(|\langle u(s) x, y\rangle-\langle x, \omega\rangle\langle\omega, y\rangle|)=0,
$$

for all $x, y \in H$ (see e.g., [6, p. 176, Proposition C.2]). Substituting $x=a \omega$ and $y=b \omega$, we obtain weak mixing of $\phi$.

$\left(3^{\prime}\right) \Rightarrow(1) . u$, defined in the proof of $(3) \Rightarrow(1)$, is equivalent to its conjugate $\bar{u}$. They are intertwined by the Tomita-Takesaki antilinear operator $J$, corresponding to $M, \omega$. Hence the proof of $(3) \Rightarrow(1)$ also shows that $\left(3^{\prime}\right) \Rightarrow(1)$. Q.E.D.

2.5. Definition. A $C^{*}$-flow $(G, A)$ is weakly mixing if $\left(G, A^{\mathrm{op}} \otimes_{*} A\right)$ is ergodic.

This is not equivalent to $\left(G, A \otimes_{*} A\right)$ ergodic. See 2.11 for an example. The choice of the minimal tensor product $\otimes_{*}$ will become clear from the proof of

2.6. Proposition. The property of weak mixing passes to factors. 
Proof. Let $(G, B)$ be a factor of the weakly mixing flow $(G, A) . B^{\mathrm{op}} \otimes_{*} B$ can be seen as a subalgebra of $A^{\mathrm{op}} \otimes_{*} A$. Since $\left(G, A^{\mathrm{op}} \otimes_{*} A\right)$ is ergodic, we obtain from [1, 3.3] that $\left(G, B^{\mathrm{op}} \otimes_{*} B\right)$ is ergodic. Q.E.D.

\subsection{Proposition. Weak mixing implies ergodicity.}

Proof. Simply note that $(G, A)$ is a factor of $\left(G, A^{\text {op }} \otimes_{*} A\right)$. Use 2.6. Q.E.D.

2.8. Proposition. Let $(G, A)$ be a $C^{*}$-flow and $\phi$ a faithful, centrally carried, $G$-invariant state. If $\phi$ is weakly mixing for $\left(G, \pi_{\phi}(A)^{\prime \prime}\right)$ then $(G, A)$ is weakly mixing ( see appendix for definition of centrally carried states).

Proof. $\phi$ is faithful on $\pi_{\phi}(A)^{\prime \prime}$. Apply 2.4 to $M=\pi_{\phi}(A)^{\prime \prime}$. We get that $\left(G, \pi_{\phi \otimes \phi}\left(A^{\mathrm{op}} \otimes_{*} A\right)^{\prime \prime}\right)$ is ergodic. By $[1,3.4]\left(G, A^{\mathrm{op}} \otimes_{*} A\right)$ is ergodic. Q.E.D.

2.9. Definition. Two $C^{*}$-flows, $(G, A)$ and $(G, B)$, are weakly disjoint if $\left(G, A^{\mathrm{op}} \otimes_{*} B\right)$ is ergodic; equivalently if $\left(G, B^{\mathrm{op}} \otimes_{*} A\right)$ is ergodic. The definitions are equivalent since $A^{\mathrm{op}} \otimes_{*} B$ and $B^{\mathrm{op}} \otimes_{*} A$ are naturally anti-isomorphic.

In particular, $(G, A)$ is weakly disjoint from itself iff it is weakly mixing. In the commutative case this definition was first given in [12].

2.10. Let $G$ be a group. A projective representation of $G$ on a Hilbert space $H$ corresponds to an action of $G$ on $\tilde{K}(H)$ (see [1]).

Proposition. If $\pi_{1}$ and $\pi_{2}$ are two projective representations of $G$, the corresponding $C^{*}$-flows are weakly disjoint iff $\bar{\pi}_{1} \otimes \pi_{2}$ is irreducible.

Proof. Let $\pi_{1}, \pi_{2}$ act on $H_{1}, H_{2}$ respectively. Identify $\left(\tilde{K}\left(H_{1}\right)\right)^{\text {op }}$ with $\tilde{K}\left(\bar{H}_{1}\right)$, and consider $\left(\tilde{K}\left(H_{1}\right)\right)^{\text {op }} \otimes_{*} \tilde{K}\left(H_{2}\right)$ as acting on $\bar{H}_{1} \otimes H_{2}$.

Now, $\tilde{K}\left(\bar{H}_{1}\right) \otimes_{*} \tilde{K}\left(H_{2}\right) \supseteq \tilde{K}\left(\bar{H}_{1} \otimes H_{2}\right)$, so if the flows are weakly disjoint, then $\left(G, \tilde{K}\left(\bar{H}_{1} \otimes H_{2}\right)\right)$ is ergodic, so by $[1,3.8] \bar{\pi}_{1} \otimes \pi_{2}$ is irreducible.

If $\bar{\pi}_{1} \otimes \pi_{2}$ is irreducible, then $\left(B\left(\bar{H}_{1} \otimes H_{2}\right)\right)_{G}=\mathbf{C}$ and by $[1,3.4]$ we get $\left(G, \tilde{K}\left(\bar{H}_{1}\right) \otimes_{*} K\left(H_{2}\right)\right)$ ergodic, so the flows are weakly disjoint. Q.E.D.

2.11. We can now see why the use of the opposite algebra in the definition of weak disjointness (in particular, weak mixing) is necessary.

Let $H$ be an infinite-dimensional Hilbert space. Let $G$ be the group of all unitaries on $H$, and $\pi$ its defining representation.

The $C^{*}$-flow $(G, \tilde{K}(H))$ is weakly mixing, since $\bar{\pi} \otimes \pi$ is irreducible. On the other hand, $\pi \otimes \pi$ is reducible, since the symmetric tensor product $(H \otimes H)_{\text {symm }}$ is an invariant subspace. So the flow $(G, \tilde{K}(H))$ is not weakly disjoint from its opposite flow.

2.12. We shall now describe several examples of weakly mixing $C^{*}$-flows.

Let a group $G$ act by continuous group automorphisms on a compact Abelian group $K$. It is well known that the flow $(G, K)$ is weakly mixing iff $G$ has no finite orbits in $\hat{K}$, the dual group of $K$, except $\{e\}$. Replacing the discrete Abelian group $\hat{K}$ by a discrete nonabelian group, we obtain a generalization:

Proposition. Let $H$ be a discrete group. Let a group $G$ act on $H$ by group automorphisms. Then $G$ acts by automorphisms on the reduced group $C^{*}$-algebra $C_{r}^{*}(H)$, and the following are equivalent:

(1) G has no finite orbits in $H$ except $\{e\}$. 
(2) $\left(G, C_{r}^{*}(H)\right)$ is an ergodic $C^{*}$-flow.

(3) $\left(G, C_{r}^{*}(H)\right)$ is a weakly mixing $C^{*}$-flow.

(4) For any action of $G$ on a discrete group $F,\left(G, C_{r}^{*}(H)\right)$ and $\left(G, C_{r}^{*}(F)\right)$ are weakly disjoint.

Proof. (1) $\Rightarrow(2)$. Let $C_{r}^{*}(H)$ act on $l^{2}(H)$ by the left regular representation, and let $W(H)$ be the weak closure of $C_{r}^{*}(H)$ in $B\left(l^{2}(H)\right)$. Since the left regular representation is faithful on $C_{r}^{*}(H)$, it is enough by $[1,3.4]$ to show that $(W(H))_{G}=$ C.

Let $a \in W(H)_{G}$ and consider $a$ as an $l^{2}$-function on $H$, which is constant on $G$-orbits. Hence $a$ must be zero on infinite orbits, or its $l^{2}$-norm would be infinite. So $a(h)=0$ for $h \neq e$ and we get $a \in \mathbf{C}$.

$(1) \Rightarrow(4) . C_{r}^{*}(H)^{\mathrm{op}} \otimes_{*} C_{r}^{*}(F)$ can be identified with $C_{r}^{*}\left(H^{\mathrm{op}} \times F\right)$. Since $G$ has no finite orbits in $H^{\mathrm{op}} \times F$, we get from (1) $\Rightarrow(2)$ that $\left(G, C_{r}^{*}\left(H^{\mathrm{op}} \times F\right)\right)$ is ergodic.

(2) $\Rightarrow(1)$. If $\left\{h_{1}, \ldots, h_{n}\right\}$ is a finite $G$-orbit in $H$, different from $\{e\}$, then $h_{1}+\cdots+h_{n} \in\left(C_{r}^{*}(H)\right)_{G}$ and $h_{1}+\cdots+h_{n} \notin \mathbf{C}$, so $\left(G, C_{r}^{*}(H)\right)$ is not ergodic.

(4) $\Rightarrow(3) \Rightarrow(2)$. Immediate. Q.E.D.

Let $G, H$ be as above. Let $\gamma$ be a multiplier (2-cocycle) on $H$, which is $G$-invariant; $\gamma\left(s h_{1}, s h_{2}\right)=\gamma\left(h_{1}, h_{2}\right)$ for all $h_{1}, h_{2} \in H$ and $s \in G$. Let $C_{r}^{*}(H, \gamma)$ be the reduced, $\gamma$-twisted, group $C^{*}$-algebra. $G$ acts by automorphisms on $C_{r}^{*}(H, \gamma)$, and we can replace $C_{r}^{*}(H)$ by $C_{r}^{*}(H, \gamma)$ everywhere in the statement and proof above.

As an example, let $H=Z^{2}$ and let

$$
\gamma_{\theta}\left(\left(m_{1}, n_{1}\right),\left(m_{2}, n_{2}\right)\right)=\exp \left(2 \pi i \theta\left(m_{1} n_{2}-m_{2} n_{1}\right)\right)
$$

where $\theta$ is real. $\gamma_{\theta}$ is a multiplier on $H$ and is invariant under the action of $G=\operatorname{sl}(2, Z)$ on $H$. We obtain an action of $G$ on the $\theta$-rotation algebra $C_{r}^{*}\left(Z^{2}, \gamma_{\theta}\right)$. If $T \in \operatorname{sl}(2, Z)$ has eigenvalues which are not roots of unity of any order, then the action of $Z$ on $C_{r}^{*}\left(Z^{2}, \gamma_{\theta}\right)$ by powers of $T$, is weakly mixing.

2.13. Let $G$ be a discrete group, $A$ a $C^{*}$-algebra. Let $A_{g}=A$ for all $g \in G$. $G$ acts on the tensor product $\otimes_{*}\left\{A_{g}: g \in G\right\}$ by $s: \otimes a_{g} \rightarrow \otimes a_{s g}$. The resulting $C^{*}$-flow will be called a Bernoulli shift.

Proposition. Any two Bernoulli shifts are weakly disjoint. In particular any Bernoulli shift is weakly mixing. Bernoulli shifts are not minimal (except for the trivial case $A=\mathbf{C}$ ).

Proof. Let $\phi$ be a nonfaithful state of $A$. Let $\phi_{g}=\phi$ for all $g \in G$. Then $\otimes\left\{\phi_{g}: g \in G\right\}$ is a $G$-invariant, nonfaithful state on $\otimes_{*}\left\{A_{g}: g \in G\right\}$. Hence shifts are not minimal.

Let $\rho_{g}$ be the universal representation of $A_{g}$. The representation $\otimes\left\{\rho_{g}: g \in G\right\}$ of $\bigotimes_{*}\left\{A_{g}: g \in G\right\}$ is faithful and covariant. By $[1,3.4]$ if we show that the $W^{*}$-flow $(G$, $\left.\otimes\left\{A_{g}^{* *}: g \in G\right\}\right)$ is ergodic, then the Bernoulli shift is also ergodic. Ergodicity of the $W^{*}$-flow is well known (see e.g. [6, p. 79]). 
Opposites and tensor products of Bernoulli shifts are also Bernoulli shifts, since

$$
\begin{gathered}
\left(\otimes_{*}\left\{A_{g}: g \in G\right\}\right) \otimes_{*}\left(\otimes_{*}\left\{B_{g}: g \in G\right\}\right) \simeq \otimes_{*}\left\{A_{g} \otimes_{*} B_{g}: g \in G\right\}, \\
\left(\otimes_{*}\left\{A_{g}: g \in G\right\}\right)^{\mathrm{op}} \simeq \otimes_{*}\left\{A_{g}^{\mathrm{op}}: g \in G\right\} .
\end{gathered}
$$

This gives the disjointness results. Q.E.D.

2.14. The free shift flow, described in $[\mathbf{1}, \mathbf{2}]$ is disjoint from all minimal flows (see 5.4) hence weakly disjoint. In particular it is weakly mixing.

\section{Hilbert-Schmidt operators defined by semicontinuous kernels.}

3.0. Let $X, Y$ be compact spaces and $\mu, \nu$ probability measures on them. If $f$ is a bounded Borel function on $X \times Y, f$ is the kernel of a Hilbert-Schmidt operator $T_{f}$ : $L^{2}(X, \mu) \rightarrow L^{2}(Y, \nu)$ defined by

$$
\left(T_{f} g\right)(y)=\int_{X} f(x, y) g(x) d \mu(x) .
$$

If $f$ is continuous, the range of $T_{f}$ contains only continuous functions. If $f$ is 1 .s.c., the range of $T_{f}$ contains linear combinations of l.s.c. functions. In this section I prove noncommutative extensions of these facts.

Let $A, B$ be $C^{*}$ algebras, acting on Hilbert spaces $H_{1}, H_{2}$ by means of representations $\pi_{1}, \pi_{2}$. Let $\Omega_{1}, \Omega_{2}$ be fixed unit vectors in $H_{1}, H_{2}$. Our "kernels" will be from $\left(A^{\mathrm{op}} \otimes_{*} B\right)^{* *} \simeq\left(A^{* *}\right)^{\text {op }} \otimes B^{* *}$.

3.1. Definition. If $c \in\left(A^{\text {op }} \otimes_{*} B\right)^{* *}$, let $T_{c}$ be the Hilbert-Schmidt operator $T_{c}$ : $H_{1} \rightarrow H_{2}$, corresponding to the vector

$$
\left(\pi_{1}^{\text {op }} \otimes \pi_{2}\right)(c)\left(\bar{\Omega}_{1} \otimes \Omega_{2}\right) \in \bar{H}_{1} \otimes H_{2} .
$$

3.2. Proposition. Let $\eta \in H_{1}$. The operator $S_{\eta}: A^{\text {op }} \otimes B \rightarrow B$ defined by

$$
S_{\eta}(a \otimes b)=\left\langle\pi_{1}(a) \eta, \Omega_{1}\right\rangle b
$$

extends to a bounded, o-weakly continuous operator $S_{\eta}:\left(A^{\mathrm{op}} \otimes_{*} B\right)^{* *} \rightarrow B^{* *}$ such that $S_{\eta}\left(A^{\mathrm{op}} \otimes_{*} B\right) \subseteq B$.

Proof. Let $\rho$ be the universal representation of $B$ acting on $H_{\rho}$. For $\xi_{1}, \xi_{2} \in H_{\rho}$,

$$
\begin{aligned}
\left\langle\rho\left(S_{\eta}(a \otimes b)\right) \xi_{1}, \xi_{2}\right\rangle & =\left\langle\pi_{1}(a) \eta, \Omega_{1}\right\rangle\left\langle\rho(b) \xi_{1}, \xi_{2}\right\rangle \\
& =\left\langle\pi_{1}^{\mathrm{op}}(a) \bar{\Omega}_{1}, \bar{\eta}\right\rangle\left\langle\rho(b) \xi_{1}, \xi_{2}\right\rangle \\
& =\left\langle\left(\pi_{1}^{\mathrm{op}} \otimes \rho\right)(a \otimes b)\left(\bar{\Omega}_{1} \otimes \xi_{1}\right), \bar{\eta} \otimes \xi_{2}\right\rangle .
\end{aligned}
$$

So for all $c \in A^{\text {op }} \otimes B$

$$
\left\langle\rho\left(S_{\eta}(c)\right) \xi_{1}, \xi_{2}\right\rangle=\left\langle\left(\pi_{1}^{\mathrm{op}} \otimes \rho\right)(c)\left(\bar{\Omega}_{1} \otimes \xi_{1}\right), \bar{\eta} \otimes \xi_{2}\right\rangle
$$

and this gives the required continuity in both norm and $\sigma$-weak topologies. Q.E.D.

3.3. Proposition. For $c \in\left(A^{\mathrm{op}} \otimes_{*} B\right)^{* *}$ and $\eta \in H_{1}$,

$$
T_{c}(\eta)=\pi_{2}\left(S_{\eta}(c)\right) \Omega_{2} \text {. }
$$


Proof. Both sides of the equality are linear and continuous in $c$ when $\left(A^{\text {op }} \otimes_{*} B\right)^{* *}$ has $\sigma$-weak topology and $H_{2}$ the weak topology. It is enough, then, to check the equality for an elementary tensor $c=a \otimes b$.

$T_{a \otimes b}$ corresponds to the vector

$$
\left(\pi_{1}^{\mathrm{op}} \otimes \pi_{2}\right)(a \otimes b)\left(\bar{\Omega}_{1} \otimes \Omega_{2}\right)=\overline{\pi_{1}\left(a^{*}\right) \Omega_{1}} \otimes \pi_{2}(b) \Omega_{2} .
$$

So,

$$
\begin{aligned}
T_{a \otimes b}(\eta) & =\left\langle\eta, \pi_{1}\left(a^{*}\right) \Omega_{1}\right\rangle \pi_{2}(b) \Omega_{2}=\left\langle\pi_{1}(a) \eta, \Omega_{1}\right\rangle \pi_{2}(b) \Omega_{2} \\
& =\pi_{2}\left(\left\langle\pi_{1}(a) \eta, \Omega_{1}\right\rangle b\right) \Omega_{2}=\pi_{2}\left(S_{\eta}(a \otimes b)\right) \Omega_{2} . \quad \text { Q.E.D. }
\end{aligned}
$$

It follows that in order to study the range of $T_{c}$ for $c$ l.s.c. we have to look at the range of the 1.s.c. elements under the operators $S_{\eta}$.

3.4. Proposition. $S_{\eta}$ is positive iff $\left\langle\eta, \pi_{1}\left(A^{+}\right) \Omega_{1}\right\rangle \geqslant 0$.

Proof. If $S_{\eta}$ is positive, let $a \in A^{+}$,

$$
\left\langle\eta, \pi_{1}(a) \Omega_{1}\right\rangle \cdot 1=S_{\eta}(a \otimes 1) \geqslant 0 .
$$

So, $\left\langle\eta, \pi_{1}\left(A^{+}\right) \Omega_{1}\right\rangle \geqslant 0$.

If $\left\langle\eta, \pi_{1}\left(A^{+}\right) \Omega_{1}\right\rangle \geqslant 0$, the linear functional on $A$ defined by $A \rightarrow\left\langle\eta, \pi_{1}(a) \Omega_{1}\right\rangle$ is positive. Let $\left(\sigma, H_{\sigma}, \omega\right)$ be the corresponding GNS objects. Let $\rho$ be the universal representation of $B$. For $\xi_{1}, \xi_{2} \in H_{\rho}$,

$$
\begin{aligned}
\left\langle\rho\left(S_{\eta}(a \otimes b)\right) \xi_{1}, \xi_{2}\right\rangle & =\left\langle\left\langle\pi_{1}(a) \eta, \Omega_{1}\right\rangle \rho(b) \xi_{1}, \xi_{2}\right\rangle \\
& =\left\langle\left\langle\overline{\sigma\left(a^{*}\right) \omega}, \bar{\omega}\right\rangle \rho(b) \xi_{1}, \xi_{2}\right\rangle \\
& =\left\langle\left(\sigma^{\text {op } \otimes \rho)}(a \otimes b)\left(\bar{\omega} \otimes \xi_{1}\right), \bar{\omega} \otimes \xi_{2}\right\rangle .\right.
\end{aligned}
$$

So for all $c \in\left(A^{\mathrm{op}} \otimes_{*} B\right)^{* *}$,

$$
\left\langle\rho\left(S_{\eta}(c)\right) \xi_{1}, \xi_{2}\right\rangle=\left\langle\left(\sigma^{\text {op }} \otimes \rho\right)(c)\left(\bar{\omega} \otimes \xi_{1}\right), \bar{\omega} \otimes \xi_{2}\right\rangle
$$

and $S_{\eta}$ is positive. Q.E.D.

3.5. Proposition. If $\Omega_{1}$ separates $\pi_{1}(A)^{\prime \prime}$ then for all $\eta \in H_{1}, S_{\eta}\left(\left(A^{\mathrm{op}} \otimes_{*} B\right)^{m}\right) \subseteq$ $\operatorname{clsp}\left(B^{m}\right)$, where clsp denotes norm closed span in $B^{* *}$.

Proof. Case 1. Let $\eta \in\left(\pi_{1}(A)^{\prime}\right)^{+} \Omega_{1}$. Then $\left\langle\eta, \pi_{1}\left(A^{+}\right) \Omega_{1}\right\rangle \geqslant 0$ so $S_{\eta}$ is positive. Since the elements of $\left(A^{\text {op }} \otimes_{*} B\right)^{m}$ are monotone limits from $A^{\text {op }} \otimes_{*} B$, and since $S_{\eta}$ is $\sigma$-weakly continuous and positive, we get $S_{\eta}\left(\left(A^{\text {op }} \otimes_{*} B\right)^{m}\right) \subseteq B^{m}$.

Case 2. Let $\eta \in \pi_{1}(A)^{\prime} \Omega_{1}$. Then $\eta$ is a linear combination of vectors in $\left(\pi_{1}(A)^{\prime}\right)^{+} \Omega_{1}$, so by Case $1, S_{\eta}\left(\left(A^{\mathrm{op}} \otimes_{*} B\right)^{m}\right) \subseteq \operatorname{sp}\left(B^{m}\right)$.

Case 3. Let $\eta \in H_{1}$ be arbitrary. Since $\Omega_{1}$ is cyclic for $\pi_{1}(A)^{\prime}, \eta$ is a norm limit of vectors in $\pi_{1}(A)^{\prime} \Omega_{1}$. By Case 2 , and since $S_{\eta}(c)$ is norm continuous in $\eta\left(\left\|S_{\eta}(c)\right\| \leqslant\right.$ $\|c\|\|\eta\|$ by the proof of 3.2), we have $S_{\eta}\left(\left(A^{\mathrm{op}} \otimes_{*} B\right)^{m}\right) \subseteq \operatorname{clsp}\left(B^{m}\right)$. Q.E.D.

3.6. Proposition. For any $c \in\left(A^{\mathrm{op}} \otimes_{*} B\right)^{* *}, T_{c}^{*}=T_{F(c)}$, where $F:\left(A^{\mathrm{op}} \otimes_{*} B\right)^{* *}$ $\rightarrow\left(B^{\mathrm{op}} \otimes_{*} A\right)^{* *}$ is the antilinear isomorphism defined by $F(a \otimes b)=b^{*} \otimes a^{*}$. 
Proof. It is enough to check the equality for $c$ an elementary tensor. Then extend by continuity and linearity as usual.

Let $a \in A, b \in B, \eta \in H_{1}, \xi \in H_{2}$.

$$
\begin{aligned}
\left\langle T_{a \otimes h}^{*} \xi, \eta\right\rangle & =\left\langle\xi, T_{a \otimes b} \eta\right\rangle=\left\langle\xi,\left\langle\pi_{1}(a) \eta, \Omega_{1}\right\rangle \pi_{2}(b) \Omega_{2}\right\rangle \\
& =\overline{\left\langle\pi_{1}(a) \eta, \Omega_{1}\right\rangle}\left\langle\xi, \pi_{2}(b) \Omega_{2}\right\rangle \\
& =\left\langle\pi_{2}\left(b^{*}\right) \xi, \Omega_{2}\right\rangle\left\langle\pi_{1}\left(a^{*}\right) \Omega_{1}, \eta\right\rangle \\
& =\left\langle\left\langle\pi_{2}\left(b^{*}\right) \xi, \Omega_{2}\right\rangle \pi_{1}\left(a^{*}\right) \Omega_{1}, \eta\right\rangle \\
& =\left\langle T_{\left(b^{*} \otimes a^{*}\right)} \xi, \eta\right\rangle . \quad \text { Q.E.D. }
\end{aligned}
$$

3.7. Proposition. For any $c \in\left(A^{\mathrm{op}} \otimes_{*} B\right)^{* *}$

$$
T_{c} \Omega_{1}=\pi_{2}\left(\left(\phi \otimes I_{B}\right)(c)\right) \Omega_{2},
$$

where $\phi$ is the state of $A$ corresponding to $\left(\pi_{1}, \Omega_{1}\right)$.

Proof. As usual, it is enough to check for $c=a \otimes b$.

$$
\begin{aligned}
T_{a \otimes b} \Omega_{1} & =\left\langle\Omega_{1}, \pi_{1}\left(a^{*}\right) \Omega_{1}\right\rangle \pi_{2}(b) \Omega_{2} \\
& =\phi(a) \pi_{2}(b) \Omega_{2}=\pi_{2}\left(\left(\phi \otimes I_{B}\right)(a \otimes b)\right) \Omega_{2} . \quad \text { Q.E.D. }
\end{aligned}
$$

\section{Weak disjointness of minimal flows.}

4.0. Proposition. Let $(G, A),(G, B)$ be minimal $C^{*}$-flows, admitting centrally carried, G-invariant states.

If the representations of $G$ on the Banach spaces $\operatorname{clsp}\left(A^{m}\right)$ and $\operatorname{clsp}\left(B^{m}\right)$ have no equivalent finite-dimensional subrepresentations, except $\mathbf{C} \subseteq A$ and $\mathbf{C} \subseteq B$, then the flows are weakly disjoint.

Proof. Let $\phi, \psi$ be centrally carried, $G$-invariant states on $A, B$ respectively. Let $\left(H_{\phi}, \pi_{\phi}, \Omega_{\phi}\right)$ and $\left(H_{\psi}, \pi_{\psi}, \Omega_{\psi}\right)$ be the corresponding GNS objects. Assume the flows are not weakly disjoint, so there is some $c \in\left(\left(A^{\mathrm{op}} \otimes_{*} B\right)^{m}\right)_{G}$ which is not constant on any dense set of states. From $\S 3$ we obtain the Hilbert-Schmidt operators $T_{c}$ : $H_{\phi} \rightarrow H_{\psi}$ and $T_{F(c)}=T_{c}^{*}: H_{\psi} \rightarrow H_{\phi}$.

Suppose the operator $T_{c}^{*} T_{c}: H_{\phi} \rightarrow H_{\phi}$ is a scalar multiple of the one-dimensional projection on $\Omega_{\phi}$. Then $T_{c}$ is a rank-one operator, with kernel orthogonal to $\Omega_{\phi}$, i.e.

$$
\left(\pi_{\phi}^{\mathrm{op}} \otimes \pi_{\psi}\right)(c)\left(\bar{\Omega}_{\phi} \otimes \Omega_{\psi}\right)=\bar{\Omega}_{\phi} \otimes T_{c}\left(\Omega_{\phi}\right)
$$

By 3.7, $T_{c}\left(\Omega_{\phi}\right)=\pi_{\psi}\left(\left(\phi \otimes I_{B}\right)(c)\right) \Omega_{\psi}$, so

$$
\left(\pi_{\phi}^{\text {op }} \otimes \pi_{\psi}\right)\left(c-\left(\phi \otimes I_{B}\right)(c)\right)\left(\bar{\Omega}_{\phi} \otimes \Omega_{\psi}\right)=0
$$

and since $\phi, \psi$ (hence $\phi \otimes \psi$ ) are centrally carried,

$$
\left(\pi_{\phi}^{\text {op }} \otimes \pi_{\psi}\right)\left(c-\left(\phi \otimes I_{B}\right)(c)\right)=0 .
$$

Now, $\phi \otimes I_{B}$ is a positive, $\sigma$-weakly continuous, $G$-equivariant map. Hence $\left(\phi \otimes I_{B}\right)(c) \in\left(B^{m}\right)_{G}$, and since $(G, B)$ is minimal, $\left(\phi \otimes I_{B}\right)(c)$ is a constant. So by the result of the preceding paragraph, $c$ is a constant on the states associated with the representation $\pi_{\phi}^{\text {op }} \otimes \pi_{\psi}$. 
Also, since $(G, A)$ and $(G, B)$ are minimal, the states $\phi$ and $\psi$ are faithful. So the representations $\pi_{\phi}, \pi_{\psi}$ and $\pi_{\phi}^{\text {op }} \otimes \pi_{\psi}$ are faithful. So the states associated with the last mentioned representation are a dense set. This contradicts the assumption that $c$ is not constant on any dense set. We deduce that $T_{c}^{*} T_{c}$ is not a multiple of the projection on $\Omega_{\phi}$.

Adding the fact that $T_{c}^{*} T_{c}$ is a positive Hilbert-Schmidt operator, we conclude that it has some positive eigenvalue $\lambda$ with a finite-dimensional eigenspace $H_{\lambda}$ different from the span of $\Omega_{\phi}$. Multiplying $c$ by $\lambda^{-1 / 2}$, we can assume that $\lambda=1$ and write $H_{1}$ for $H_{\lambda}$. Let $H_{1}^{\prime}$ be the 1-eigenspace of $T_{c} T_{c}^{*}$ on $H_{\psi}$. We see immediately that $T_{c} H_{1} \subseteq H_{1}^{\prime}$ and $T_{c}^{*} H_{1}^{\prime} \subseteq H_{1}$. Since $T_{c}^{*} T_{c}=1$ on $H_{1}$ and $T_{c} T_{c}^{*}=1$ on $H_{1}^{\prime}$, we conclude that $T_{c}$ is an isometry of $H_{1}$ onto $H_{1}^{\prime}$. Since $c$ is $G$-invariant we obtain that $H_{1}, H_{1}^{\prime}$ are $G$-invariant subspaces of $H_{\phi}, H_{\psi}$ and $T_{c}$ gives an equivalence of the representations of $G$ on $H_{1}$ and $H_{1}^{\prime}$.

Now I will find subspaces $D \subseteq \operatorname{clsp}\left(A^{m}\right)$ and $D^{\prime} \subseteq \operatorname{clsp}\left(B^{m}\right)$ such that $D$ has a representation of $G$ equivalent to $H_{1}$ and $D^{\prime}$ equivalent to $H_{1}^{\prime}$. Let

$$
D=\left\{S_{T_{c}(\eta)}(F(c)): \eta \in H_{1}\right\}, \quad D^{\prime}=\left\{S_{T_{*}^{*}(\xi)}(c): \xi \in H_{1}^{\prime}\right\}
$$

We get $D \subseteq \operatorname{clsp}\left(A^{m}\right)$ and $D^{\prime} \subseteq \operatorname{clsp}\left(B^{m}\right)$ from 3.5. The map $L: H_{1} \rightarrow D$ defined by $L(\eta)=S_{T_{c}(\eta)}(F(c))$, is $G$-equivariant by invariance of $c$ and $F(c)$, onto by definition of $D$, and $1-1$ since

$$
\begin{aligned}
\pi_{\phi}(L \eta) \Omega_{\phi} & =\pi_{\phi}\left(S_{T_{c}(\eta)}(F(c))\right) \Omega_{\phi} \\
& =T_{F(c)}\left(T_{c} \eta\right)=T_{c}^{*} T_{c} \eta=\eta,
\end{aligned}
$$

using 3.3 in the second equality. A similar proof works for $H_{1}^{\prime}$ and $D^{\prime}$.

We are done now, since $D, D^{\prime}$ give equivalent, finite-dimensional subrepresentations, and $D \neq \mathbf{C}$ since $\pi_{\phi}(D) \Omega_{\phi}=H_{1} \neq \operatorname{span}\left(\Omega_{\phi}\right)$. Q.E.D.

4.1. Proposition. Let $(G, A),(G, B)$ be weakly disjoint $C^{*}$-flows. The only equivalent, finite-dimensional subrepresentations of $A, B$ are $\mathbf{C} \subseteq A$ and $\mathbf{C} \subseteq B$.

Proof. Let $D_{1}, D_{2}$ be equivalent, finite-dimensional, irreducible subspaces of $A$ and $B$. Assume $D_{1} \neq \mathbf{C}$. Then by irreducibility $1 \notin D_{1}$. As in the beginning of the proof of $[1,4.7]$, let $\left\{e_{1}, \ldots, e_{n}\right\}$ be a base of $D_{1}$, orthonormal with respect to a $G$-invariant inner product. Let $L: D_{1} \rightarrow D_{2}$ be a $G$-equivalence and let

$$
c=\sum_{i=1}^{n} e_{i}^{*} \otimes L e_{i},
$$

considered as an element of $A^{\mathrm{op}} \bigotimes_{*} B$. An elementary computation shows that $c$ is $G$-invariant, so a constant. $\sum_{i=1}^{n} e_{i}^{*} \otimes L e_{i}=\alpha \otimes 1$ for some $\alpha \in \mathbf{C}$. This implies that $\left\{e_{1}^{*}, \ldots, e_{n}^{*}, \alpha\right\}$ are linearly dependent. This is a contradiction since $\left\{e_{1}^{*}, \ldots, e_{n}^{*}\right\}$ are linearly independent and do not span 1. Q.E.D. 
4.2. Theorem. Let $(G, A)$ and $(G, B)$ be minimal $C^{*}$-flows, admitting centrally carried, G-invariant states.

The flows are weakly disjoint iff $A, B$ have no equivalent, finite-dimensional subrepresentations except $\mathbf{C} \subseteq A$ and $\mathbf{C} \subseteq B$.

In particular, $(G, A)$ is weakly mixing iff $A$ has no finite-dimensional subrepresentation except $\mathbf{C} \subseteq A$.

Proof. Elements of $\operatorname{clsp}\left(A^{m}\right)$ have a comeager set of continuity points in the set of states. The theorem is obtained by combining 4.0, 4.1 and [1, 4.7]. Q.E.D.

Corollary 1. Let $(G, A),(G, B)$ be as in the theorem. They are weakly disjoint iff $\left(G, A^{\mathrm{op}}\right)$ and $(G, B)$ are weakly disjoint, iff $\left(G, A_{\mathrm{ap}}\right)$ and $\left(G, B_{\mathrm{ap}}\right)$ are weakly disjoint.

Corollary 2. Let $(G, A)$ be as in the theorem. $(G, A)$ is weakly mixing iff $A_{\text {ap }}=\mathbf{C}$.

Proof (of Corollary 2). By Corollary 1. $(G, A)$ is weakly mixing iff $\left(G, A_{\text {ap }}\right)$ is. So it is enough to prove the corollary in the case that $(G, A)$ is almost periodic, otherwise replace $A$ by $A_{\text {ap }}$. Without limiting generality we can assume that $G$ is compact.

Now if $(G, A)$ is weakly mixing, then $A$ has no finite-dimensional invariant subspaces. But since $G$ is compact, finite-dimensional invariant subspaces span a dense subspace of $A$, hence $A=\mathbf{C}$. Conversely if $A_{\text {ap }}=\mathbf{C}$ then $A=A_{\text {ap }}=\mathbf{C}$ and $(G, A)$ is weakly mixing. Q.E.D.

4.3. Notes. (1) The proof of the main theorem follows an idea, first used in [3] to show the existence of eigenfunctions for a distal flow, and later developed in $[\mathbf{8}, \mathbf{1 2}]$ to give the commutative case of our theorem.

(2) In the commutative case of the main theorem, we have to require that the flows have invariant measures. The assumption of centrally carried is, of course, satisfied auotmatically.

(3) If $A$ has a trace, and $G$ is amenable, $A$ has a $G$-invariant trace, so there exists a $G$-invariant centrally carried state on $A$.

\section{Disjointness.}

5.0. The definition of weak disjointness in topological dynamics is motivated by the important concept of disjointness. Disjointness of flows implies that they have no nontrivial equivalent factors, but is easier to handle than that property. In $C^{*}$-flows disjointness does not have the same property, since a $C^{*}$-flow may be disjoint from itself (see 5.4). Still, I include here a definition of disjointness and some elementary properties.

5.1. Definition. Let $(G, A),(G, B)$ be $C^{*}$-flows. The flows are disjoint if for all $G$-invariant, proper hereditary subalgebras $J$ of $A^{\text {op }} \otimes_{*} B$, if $J \cap A^{\text {op }}=0, J \cap B=0$, then $J=0$. The definition is symmetric in $A$ and $B$ for the same reason as in the definition of weak disjointness.

In the commutative case, this reduces to the definition given by [4, Lemma II.1].

If $A^{\text {op }} \otimes_{\alpha} B$ is some bigger $C^{*}$-tensor product of $A^{\text {op }}$ and $B$, let $J$ be the kernel of the natural homomorphism $A^{\text {op }} \otimes_{\alpha} B \rightarrow A^{\text {op }} \otimes_{*} B$. Now $J$ is $G$-invariant and $J \cap A^{\text {op }}$ $=0, J \cap B=0$ but $J \neq 0$. Thus, the definition of disjointness cannot be satisfied 
with a bigger tensor product. This is the reason for choosing $\otimes_{*}$ in the definition.

\subsection{Proposition. Of two disjoint flows, at least one is minimal.}

Proof. Let $(G, A),(G, B)$ be disjoint $C^{*}$-flows. Let $J_{1} \subseteq A$ and $J_{2} \subseteq B$ be proper, $G$-invariant hereditary subalgebras. Consider $J=J_{1}^{\text {op }} \otimes_{*} J_{2}$ as a proper, $G$ invariant, hereditary subalgebra of $A^{\mathrm{op}} \otimes_{*} B$. Let $\phi$ be a state of $A$ which is zero on $J_{1}$, and let $\psi$ be any state of $B$. Then $\phi \otimes \psi$ is zero on $J$.

Now let $1 \otimes b \in J \cap B$. Then $\psi(b)=(\phi \otimes \psi)(1 \otimes b)=0$. Since $\psi$ is arbitrary, $b=0$ and so $J \cap B=0$. Similarly $J \cap A^{\text {op }}=0$. By disjointness $J=0$, that is $J_{1}=0$ or $J_{2}=0$. One of the flows is minimal. Q.E.D.

5.3. Proposition. Let $(G, A),(G, B)$ be minimal $C^{*}$-flows. The flows are disjoint iff $\left(G, A^{\mathrm{op}} \otimes_{*} B\right)$ is minimal.

Proof. In the definition, $J \cap A^{\text {op }}=0$ and $J \cap B=0$ are automatically satisfied, because of minimality, so the definition just says that every proper, $G$-invariant, hereditary subalgebra of $A^{\mathrm{op}} \otimes_{*} B$ is zero. Q.E.D.

COROLlary. For minimal flows, disjointness implies weak disjointness.

5.4. Proposition. Let $G, \pi_{1}, \pi_{2}$ be as in 2.10 . The flows $\left(G, \tilde{K}\left(H_{1}\right)\right)$ and $\left(G, \tilde{K}\left(H_{2}\right)\right)$ are disjoint iff one of $\pi_{1}, \pi_{2}$ is irreducible, finite dimensional, and

$$
\left(\bar{\pi}_{1} \otimes \pi_{2}\right)(G)^{\prime}=\bar{\pi}_{1}(G)^{\prime} \otimes \pi_{2}(G)^{\prime} .
$$

Proof. One of two disjoint $C^{*}$-flows must be minimal, say $\left(G, \tilde{K}\left(H_{1}\right)\right)$. So what we have to prove is: if $\pi_{1}$ is irreducible, finite dimensional, then $\left(G, K\left(H_{1}\right)\right)$ and $\left(G, \tilde{K}\left(H_{2}\right)\right)$ are disjoint iff $\left(\bar{\pi}_{1} \otimes \pi_{2}\right)(G)^{\prime}=\mathbf{C} \otimes \pi_{2}(G)^{\prime}$.

First assume that $\left(\bar{\pi}_{1} \otimes \pi_{2}\right)(G)^{\prime}=\mathbf{C} \otimes \pi_{2}(G)^{\prime}$. Every $G$-invariant projection in $\bar{H}_{1} \otimes H_{2}$ is of the form $1 \otimes p$, where $p$ is a $G$-invariant projection in $H_{2}$. Then every $G$-invariant subspace of $\bar{H}_{1} \otimes H_{2}$ is of the form $\bar{H}_{1} \otimes V$, where $V$ is a $G$-invariant subspace of $\mathrm{H}_{2}$. The $G$-invariant, hereditary subalgebras of $K\left(\bar{H}_{1}\right) \otimes_{*} \tilde{K}\left(H_{2}\right)$ are of the form $K\left(\bar{H}_{1}\right) \otimes_{*} K(V)^{*}$ or $K\left(\bar{H}_{1}\right) \otimes \tilde{K}(V)$. The only one of these that has zero intersection with $\tilde{K}\left(H_{2}\right)$ is $K(\bar{H}) \otimes_{*} K(0)=0$. The flows are disjoint by definition.

Now assume the flows are disjoint. Let $p \neq 0,1$ be a projection in $\left(\bar{\pi}_{1} \otimes \pi_{2}\right)(G)^{\prime}$, and let $V=p\left(\bar{H}_{1} \otimes H_{2}\right)$ be the corresponding invariant subspace. $K(V)$ is a nonzero, $G$-invariant, hereditary subalgebra of $K\left(\bar{H}_{1}\right) \otimes \tilde{K}\left(H_{2}\right)$, and $K(V) \cap K\left(\bar{H}_{1}\right)$ $=0$ because of the irreducibility of $\pi_{1}$. Note that $K(V) \cap K\left(\bar{H}_{1}\right)=K\left(\bar{H}_{1}\right)$ is impossible since $1 \notin K(V)$. By disjointness, $K(V) \cap \tilde{K}\left(H_{2}\right) \neq 0$. Let $T \in \tilde{K}\left(H_{2}\right)$ such that $1 \otimes T \in K(V)$. Then $1 \otimes T^{*} T \in K(V)$ and so $1 \otimes q \in K(V)$ for some projection $q \neq 0$. We conclude that $V$ contains a subspace of the form $H_{1} \otimes W$, $W \neq 0$, and, of course, we may take $W$ to be $G$-invariant.

Now consider the $W^{*}$-algebras $\mathbf{C} \otimes \pi_{2}(G)^{\prime} \subseteq\left(\bar{\pi}_{1} \otimes \pi_{2}\right)(G)^{\prime}$. We have shown that every nonzero projection in the bigger algebra contains a nonzero projection in the smaller algebra. A straightforward Zornification shows that the algebras are equal. Q.E.D.

5.5. Shift automorphisms of free products were constructed in [2] and discussed further in [1]. One concrete example is the automorphism of $C_{r}^{*}\left(F_{\infty}\right)$ obtained by a 
free permutation of the generators. ( $F_{\infty}$ is the free group on infinitely many generators.)

Proposition. Let $(G, A)$ be a minimal shift flow where $G$ is amenable. Then $(G, A)$ is disjoint from all minimal flows (including itself).

Proof. Let $\phi$ be the free product state on $A$. In the example above this is the standard trace. By $[2,5.1]$ if $(G, B)$ is any $C^{*}$-flow the $G$-invariant states of $\left(G, A \otimes_{*} B\right)$ are exactly $\{\phi \otimes \psi: \psi$ is $G$-invariant on $B\}$.

Now let $(G, B)$ be minimal. Any $\psi$ which is $G$-invariant on $B$ is faithful. So $\phi \otimes \psi$ is faithful on $A \otimes_{*} B$ (see [2, Appendix]). By [1, 4.3] $\left(G, A \otimes_{*} B\right)$ is minimal so the flows are disjoint. Q.E.D.

\section{Appendix.}

6.1. Proposition. Let $M$ be a $W^{*}$-algebra, $\phi$ a normal state on $M, \pi_{\phi}$ the corresponding representation of $M$.

$\phi$ is faithful on $\pi_{\phi}(M)$ iff the carrier projection of $\phi$ is central.

PROOF. $\phi$ is faithful on $\pi_{\phi}(M)$ iff for all $a \in M$

$$
\phi\left(\pi_{\phi}\left(a^{*} a\right)\right)=0 \Rightarrow \pi_{\phi}\left(a^{*} a\right)=0 .
$$

I.e., iff for all $a, b \in M, \phi\left(a^{*} a\right)=0 \Rightarrow \phi\left(b^{*} a^{*} a b\right)=0$. In other words: the left ideal $L_{\phi}=\left\{a \in M: \phi\left(a^{*} a\right)=0\right\}$ is a two-sided ideal. This means exactly that the carrier projection of $\phi$ is central. Q.E.D.

6.2. Proposition. (i) Tensor products of centrally carried states are centrally carried.

(ii) A state which is invariant by a large [11, 7.12] group of automorphisms is centrally carried.

(iii) A trace is centrally carried.

Proof. (i) If $\phi_{i}$ are faithful on $\pi_{\phi_{i}}\left(M_{i}\right)$ for $i=1,2$ then $\phi_{1} \otimes \phi_{2}$ is faithful on $\pi_{\phi_{1}}\left(M_{1}\right) \otimes \pi_{\phi_{2}}\left(M_{2}\right)$ (see $\left.[14,5.12]\right)$. This is naturally isomorphic to $\pi_{\phi_{1} \otimes \phi_{2}}\left(M_{1} \otimes M_{2}\right)$.

(ii) Let $G$ be a large group of automorphisms of $M$. Let $e$ be the largest $G$-finite projection in $M$. Then $M_{G} e \subseteq \operatorname{center}(M)[6$, p. 59]. If $\phi$ is a $G$-invariant state and $p$ its carrier projection, then $p$ is $G$-invariant, and $p \leqslant e$. Hence $p$ is central.

(iii) A trace is invariant under the large group of inner automorphisms. Q.E.D.

6.3. Proposition. Let $A$ be a $C^{*}$-algebra, $\phi$ a state on $A$. $\phi$ is faithful on $\pi_{\phi}(A)^{\prime \prime}$ iff the carrier projection of $\phi$ is central in $A^{* *}$. If $\phi$ is invariant under a large group of automorphisms then $\phi$ is centrally carried.

Proof. Apply 6.1 and 6.2 to $M=A^{* *}$. Q.E.D.

\section{REFERENCES}

1. D. Avitzour, Noncommutative topological dynamics. I, Trans. Amer. Math. Soc. 282 (1984), 109-119.

2. Free products of $C^{*}$-algebras, Trans. Amer. Math. Soc. 271 (1982), 423-435.

3. H. Furstenberg, The structure of distal flows, Amer. J. Math. 85 (1963), 477-515.

4. Disjointness in ergodic theory minimal sets and a problem in Diophantine approximation, Math. Systems Theory 1 (1967), 1-49.

5. W. L. Green, Topological dynamics and $C^{*}$-algebras. Trans. Amer. Math. Soc. 210 (1975), 107-121. 
6. A. Guichardet, Systemes dynamiques non commutatifs, Soc. Math. France, Paris, 1974.

7. H. B. Keynes and J. B. Robertson, On ergodicity and mixing in topological transformation groups, Duke. Math. J. 35 (1968), 809-819.

8. Eigenvalue theorems in topological transformation groups, Trans. Amer. Math. Soc. 139 (1969), 359-369.

9. D. Laison and G. Laison, Topological dynamics on $C^{*}$-algebras, Trans. Amer. Math. Soc. 204 (1975), 197-205.

10. D. Oleson, G. K. Pedersen and M. Takesaki, Ergodic actions of compact Abelian groups, Copenhagen Preprint Series No. 19, 1979.

11. G. K. Pedersen, $C^{*}$-algebras and their automorphism groups, London Math. Soc. Monographs, no. 14, Academic Press, London and New York, 1979.

12. R. Peleg, Weak disjointness of transformation groups, Proc. Amer. Math. Soc. 33 (1972), 165-174.

13. J. Dixmier, Les $C^{*}$-algèbres et leurs représentations, Gauthier-Villars, Paris, 1964.

14. M. Takesaki, Theory of operator algebras, Springer-Verlag, New York, 1979.

Department of Mathematics, University of California, Berkeley, California 94720

Current address: Elta Electronics Industries, P.O. Box 330, Ashdod, Israel 